\title{
Expression of intelectin-1 in bronchial epithelial cells of asthma is correlated with T-helper 2 (Type-2) related parameters and its function
}

Taiji Watanabe ${ }^{1}$, Kazuyuki Chibana ${ }^{1 *}$, Taichi Shiobara ${ }^{1}$, Rinna Tei ${ }^{1}$, Ryosuke Koike ${ }^{1}$, Yusuke Nakamura ${ }^{1}$, Ryo Arai ${ }^{1}$, Yukiko Horigane', Yasuo Shimizu', Akihiro Takemasa', Takeshi Fukuda², Sally E. Wenzel ${ }^{3}$ and Yoshiki Ishii ${ }^{1}$

\begin{abstract}
Background: Intelectin-1 (ITLN-1) is secreted by intestinal goblet cells and detectable in blood. Its expression is increased in IL-13-overexpressing mouse airways. However, its expression and function in human airways is poorly understood.

Methods: Distal and proximal bronchial epithelial cells (BECs) were isolated from bronchoscopic brushings of disease control (D-CON), COPD, inhaled corticosteroid-treated asthma (ST-Asthma) and inhaled corticosteroid-naïve asthma (SN-Asthma) patients. ITLN-1 mRNA expression in freshly isolated BECs, primary cultured BECs with or without IL-13 and inhibition effects of mometasone furoate (MF) were investigated by quantitative real-time PCR (qPCR). Correlations between ITLN-1 mRNA and Type-2 related parameters (e.g. FeNO, IgE, iNOS, CCL26, periostin and DPP4 mRNA) were analyzed. ITLN-1 protein distribution in asthmatic airway tissue was assessed by immunohistochemistry. Bronchial alveolar lavage (BAL) and serum ITLN-1 protein were measured by ELISA. The effect of recombinant human (rh) ITLN-1 on stimulated production of CXCL10 and phospho(p)-STAT1 expression examined in lung fibroblasts.
\end{abstract}

Results: ITLN-1 mRNA was expressed in freshly isolated BECs and was correlated with Type-2 related parameters. ITLN-1 protein was increased in goblet cells in SN-Asthmatics and increased in SN-Asthmatic BAL fluid. There were no any differences in serum ITLN-1 concentration between ST and SN-Asthma. IL-13 enhanced ITLN-1 expression and inhibited by MF from BECs in vitro, while rhITLN-1 inhibited CXCL10 production and p-STAT1 expression in HFL-1 cells.

Conclusion: ITLN-1 is induced by IL-13 and expressed mainly in goblet cells in untreated asthma where its levels correlate with known Type-2 related parameters. Further, ITLN-1 inhibits Type-1 chemokine expression.

Keywords: Intelectin-1, Bronchial asthma, Bronchial epithelial cells, IL-13, Type-2 related parameters

\section{Background}

Asthma affects nearly 300 million people worldwide but is a heterogeneous disorder comprised of different inflammatory characteristics. Type-2 cytokines (specifically, interleukin (IL)-4, IL-5, and IL-13) are known to play a substantial pathobiological role in many cases.

\footnotetext{
*Correspondence: kchibana@dokkyomed.ac.jp

1 Department of Pulmonary Medicine and Clinical Immunology, Dokkyo

Medical University School of Medicine, Tochigi, Japan

Full list of author information is available at the end of the article
}

These cytokines, including IL-13 contribute to a Type2-high molecular asthma phenotype in about $50 \%$ of patients with asthma, and are widely believed to play important roles in asthma pathophysiology [1-7]. Furthermore, IL-13-induced periostin [8] and DPP4 can be measured in peripheral blood and are used as biomarkers to predict the efficacy of anti-IL-13 antibodies in human asthma patients [9-11].

Intelectin-1 (ITLN-1) was cloned in 1998 by Komiya et al. from the murine intestinal tract [12]. Human ITLN-1 is a prophylactic soluble lectin discovered that recognizes 
galactofuranose in the bacterial cell wall [13]. The expression of ITLN-1 in the gastrointestinal tract is strongly induced by parasitic infections $[14,15]$, suggesting that it is associated with prophylaxis in the gastrointestinal tract. ITLN-1 has been primarily studied in the gastrointestinal tract where it is expressed in intestinal goblet cells, primarily from fetal small intestine. It is detected in blood and can be measured intraluminally as well [16]. ITLN-1 is increased in the airways of IL-13-overexpressing mice, where it appears to be a protein component of mucus associated with intense eosinophilic airway inflammation [17, 18]. However, its expression and role in human asthmatic airways is poorly understood. ITLN-1 was also reported as one of the adipocytokine with anti-inflammatory effects [19]. CXCL10 is a chemokine that attracts T-helper (Th)1 cells $[20]$ and strongly induced by IFNy. When viral infection occurs, viral recognition receptors, such as Toll-like receptor 3 (TLR3) expressed on BECs, are activated to produce inflammatory cytokines and chemokines, including CXCL10 [21]. Autocrine activation of interferon (IFN) receptors further activates Janus kinase-Signal Transducers and Activator of Transcription (JAK-STAT) signaling pathway, promoting an antiviral state. Moreover, fibroblast like cell produce type I IFN and CXCL10 after stimulation with double stranded RNA [22], perhaps contributing to the pathogenesis of viral infections. Little knowledge exists concerning how the fibroblasts respond to ITLN-1 and which signaling pathways might be involved.

We hypothesized that whether ITLN-1 was induced by IL-13 and correlated to type- 2 related markers and inhibited Th1 signaling pathway. In this study, we evaluated ITLN-1 mRNA and protein expression in airway cells, tissue and fluid from asthma, COPD, and disease control subjects obtained via bronchoscopy. BAL and serum ITLN-1 levels were also measured. We compared expression of ITLN-1 mRNA with various Type- 2 related parameters. Finally, we investigated a possible function of ITLN-1 in the airways.

\section{Methods}

\section{Study population}

We conducted a retrospective study of 61 patients who visited the Department of Pulmonary Medicine and Clinical Immunology of Dokkyo Medical University
Hospital from June 2009 to March 2014 (Table 1). Bronchial brushings were performed to analyze the expression levels of ITLN-1 mRNA. Transbronchial lung biopsy (TBLB) and endobronchial biopsy (EBB) were performed. All subjects met the American Thoracic Society criteria for asthma and had a pre-bronchodialator FEV1 greater than $80 \%$ of predicted with an FEV1/FVC greater than $70 \%$. The ST-Asthma group was regularly treated with inhaled corticosteroids (ICS), while the Steroid Naïve (SN)-Asthma group had symptoms, such as cough with wheezing and night time dyspnea, but had not been treated with ICS or oral corticosteroid (OCS) for at least 6 months. Patients were defined as having COPD if the forced expiratory volume in $1 \mathrm{~s}$ (FEV1)/forced vital capacity (FVC) (FEV1/FVC) was $<70 \%$ with fixed bronchial obstruction after bronchodilator. Disease control subjects (D-CON) were defined as those without asthma/ COPD who had undergone bronchoscopy because of abnormal chest X-ray shadows. Lung cancer was found in most of D-CON and COPD patients by bronchoscopy. D-CON (as opposed to healthy control) participants were studied, as research bronchoscopy on healthy individuals is not allowed in Japan. Written informed consent was obtained from all participants to perform the procedure and utilize extra tissue/cells for research purposes. This study was approved by the Ethics Committee of Dokkyo Medical University School of Medicine (hop-m22095).

\section{Bronchoscopy with bronchial epithelial cell brushing}

Bronchial brushings were performed with a standard, sterile, single-sheathed nylon cytology brush (Olympus T-260; Olympus, Tokyo, Japan). A total of 4 brushings were performed in the distal and proximal airways. Distal bronchial epithelial cells (BECs) were obtained from airways situated about $1 \mathrm{~cm}$ away from the pleura, as identified by X-ray guidance [7]. Proximal BECs were collected by scraping directly from the second carina. TBLB and EBB were available from a small number of participants for ITLN-1 expression by immunohistochemistry. We did not collect bronchial alveolar lavage (BAL) and serum samples at the beginning of this study. Given the results of microarray study [7] that strongly expressed ITLN-1 mRNA in SN-Asthma as well as IL-13 stimulated cells, we decided to accumulate serum and BAL samples

Table 1 Total subjects in this study

\begin{tabular}{llllllllll}
\hline & N & Age & M:F & FEV1/FVC (\%) & FEV1 (\%) & FeNO (ppb) & ICS $(\boldsymbol{\mu g})$ & OCS use & Smoker (N:E:C) \\
\hline D-CON & 13 & $61 \pm 5^{* * *}$ & $11: 2$ & $78 \pm 2$ & $93 \pm 3$ & $27 \pm 3$ & 0 & 0 & $4: 8: 1$ \\
COPD & 17 & $72 \pm 2^{*}$ & $14: 3$ & $50 \pm 4^{*}$ & $57 \pm 6$ & $32 \pm 7$ & 0 & 0 & $1: 7: 9$ \\
ST-Asthma & 13 & $51 \pm 4$ & $10: 3$ & $73 \pm 5$ & $88 \pm 6$ & $45 \pm 5$ & $723 \pm 86$ & 2 & $3: 8: 2$ \\
SN-Asthma & 18 & $48 \pm 4$ & $13: 5$ & $73 \pm 3$ & $83 \pm 3$ & $129 \pm 2^{*}$ & 0 & 0 & $6: 10: 2$ \\
\hline
\end{tabular}

${ }^{*} p<0.0001$ vs ST or SN-Asthma, ${ }^{* * *} p<0.05$ vs other groups 
from asthma patients subsequently. Some cases were not able to collect BAL samples because of severe cough or hypoxemia. Finally, total 18 subjects (5 ST-Asthma and $13 \mathrm{SN}$-Asthma) were able to collect BAL and 16 subjects (6 ST-Asthma and $10 \mathrm{SN}$-Asthma) serum samples (Tables 2,3). Table 4 shows 3 subjects who were received bronchoscopy pre and post ICS-treatment.

\section{Quantitative real-time PCR}

Expression of ITLN-1, iNOS, CCL26, periostin and DPP4 mRNA in BECs and the expression of CXCL10 mRNA in HFL-1 cells were following reverse transcription (RT), and then real-time quantitative SYBR Green fluorescent PCR, as described previously [2, 3, 7]. First-strand cDNA was synthesized using the PrimeScript RT reagent Kit (Takara Bio Inc., Shiga, Japan) with both oligo (dT) primers and random hexamers. Reverse transcription was performed with a Takara PCR Thermal Cycler MP (TP3000). The following are the primer sequences used for amplification of ITLN-1, iNOS, CCL26, periostin, DPP4, CXCL10, and GAPDH: ITLN-1: forward primer, TGAGGGTCACCGGATGTAAC, reverse primer, GGACTGGCCTCTGGAAAGTA. iNOS: forward primer, GACCAGTACGTTTGGCAATG, reverse primer, TTTCAGCATGAAGAGCGATTT. CCL26: forward primer, GCTGCTTCCAATACAGCCACA, reverse primer, TCCTTGGATGGGTACAGACTTTC. periostin: forward primer, TGTTGCCCTGGTTATATGAGAA, reverse primer, ACATGGTCAATGGGCAAAAC. DPP4: forward primer, GCACGGCAACACATTGAA, reverse primer, TGAGGTTCTGAAGGCCTAAATC. CXCL10: forward primer, GAAAGCAGTTAGCAAGGAAAGGT, reverse primer, GACATATACTCCATGTAGGGAAGTGA. GAPDH: forward primer, GCACCGTCAAGGCTGAGAAC, reverse primer, TGGTGAAGACGCCAGTGGA. B2M: forward primer, TTCTGGCCTGGAGGCTATC, reverse primer, TCAGGAAATTTGACTTTCCATTC. RPLPO: forward primer, TCTACAACCCTGAAGTGCTTGAT, reverse primer, CAATCTGCAGACAGACACTGG.

The $12.5 \mu \mathrm{L}$ PCR contained $2 \mu \mathrm{L}$ of cDNA template, $25 \mu \mathrm{M}$ in $0.5 \mu \mathrm{L}$ each forward and reverse primers and $6.25 \mu \mathrm{L}$ of SYBR Premix Ex Taq (Takara). GAPDH was evaluated by using the same PCR protocol as for the interest genes-related pathway elements. DNA was amplified for 40 cycles via denaturation for $5 \mathrm{~s}$ at $95{ }^{\circ} \mathrm{C}$ and annealing for $30 \mathrm{~s}$ at $60{ }^{\circ} \mathrm{C}$, using the Takara Thermal Cycler Dice (TP900). PCR assays were performed and analyzed using the Thermal Cycler Dice Real Time System version 4.2 (Takara Bio Inc). The specificity of the reactions was determined by melting curve analysis. The relative expression of each gene of interest and GAPDH were calculated using the $\Delta \Delta \mathrm{Ct}$ method.

\section{Correlations between Type- 2 related parameters and ITLN-1 expression}

FeNO was measured before bronchoscopy at a flow rate of $50 \mathrm{~mL} / \mathrm{s}$ using the nitric oxide analyzer (NOA) $280 \mathrm{i}^{\circledR}$ (Sievers, CO). Correlations between FeNO, serum IgE (measured in the hospital's clinical lab.) and ITLN-1

Table 2 Subjects for analysis of BAL ITLN-1 protein

\begin{tabular}{llllllr}
\hline & N & Age & M:F & FEV1/FVC (\%) & \%FEV1 (\%) & FeNO (ppb) \\
\hline ST-Asthma & 5 & $53 \pm 4$ & $4: 1$ & $81 \pm 3$ & $90 \pm 8$ & $53 \pm 11$ \\
SN-Asthma & 13 & $51 \pm 4$ & $10: 3$ & $71 \pm 3$ & $82 \pm 4$ & $124 \pm 23$ \\
\hline
\end{tabular}

Table 3 Subjects for analysis of serum ILTN-1 protein

\begin{tabular}{|c|c|c|c|c|c|c|c|}
\hline & $\mathbf{N}$ & Age & $\mathrm{M}: \mathrm{F}$ & FEV1/FVC (\%) & \%FEV1 (\%) & FeNO (ppb) & ICS $(\mu g)$ \\
\hline ST-Asthma & 6 & $53 \pm 6$ & $3: 3$ & $80 \pm 5$ & $96 \pm 6$ & $42 \pm 31$ & $800 \pm 120$ \\
\hline SN-Asthma & 10 & $43 \pm 5$ & $8: 2$ & $77 \pm 4$ & $86 \pm 5$ & $147 \pm 24^{* *}$ & 0 \\
\hline Healthy control & 8 & $25 \pm 1^{* *}$ & $5: 3$ & ND & ND & $19 \pm 2$ & 0 \\
\hline
\end{tabular}

** $p<0.01$ vs other groups

Table 4 Subjects for analysis of ILTN-1 mRNA pre or post ICS treatment

\begin{tabular}{|c|c|c|c|c|c|c|c|}
\hline & N (distal: proximal) & Age & $\mathrm{M}: \mathrm{F}$ & FEV1/FVC (\%) & \%FEV1 (\%) & FeNO (ppb) & ICS ( $\mu \mathrm{g})$ \\
\hline ST-Asthma (post treatment) & $6(3: 3)$ & $65 \pm 4$ & $3: 0$ & $69 \pm 4$ & $84 \pm 4$ & $51 \pm 4$ & 400 \\
\hline SN-Asthma (pre treatment) & $6(3: 3)$ & $65 \pm 4$ & $3: 0$ & $68 \pm 4$ & $89 \pm 4$ & $171 \pm 49^{* * *}$ & 0 \\
\hline
\end{tabular}

***p $<0.05$ vs ST-Asthma 
mRNA expression in distal and proximal BECs from both ST and SN-Asthma subjects were analyzed. We also measured correlations between ITLN-1 mRNA and iNOS, CCL26, periostin and DPP4 mRNA in distal and proximal BECs from the same subjects.

\section{Immunohistochemistry}

Transbronchial lung biopsies (TBLB) and EBB from SNAsthma, and ST-Asthma subjects were fixed in formalin. Serial $4 \mu \mathrm{m}$ sections were immunostained using a rabbit polyclonal antibody against ITLN-1 (1:500) (Abcam, MA) with Dako EnVisionTM FLEX Mini Kit High pH detection system including secondary anti-rabbit antibody for detection. Data were collected using an all-in-one fluorescence microscope, BZ-X700 (KEYENCE, Tokyo, Japan).

\section{Quantification of ITLN-1 and CXCL10 protein by ELISA}

BAL fluid from 18 asthma subjects, 5 ST-Asthma and 13 SN-Asthma (Table 2) and serum from 6 ST-Asthma, and 10 SN-Asthma subjects (Table 3) was collected. There was a little overlap in three study groups. Cell culture supernatants were performed on ALI cultured BECs and HFL-1 cells. ITLN-1 (Immuno-Biological Laboratories Co., Gunma, Japan) or CXCL10 (R\&D Systems, Minneapolis, MN) were measured by commercial sandwich ELISAs. Assay ranges are $0.31-20 \mathrm{ng} / \mathrm{mL}$ for ITLN-1 and $7.8-500 \mathrm{pg} / \mathrm{mL}$ for CXCL10, respectively.

\section{Culture methods for primary BECs and HFL-1 cells}

Freshly isolated BECs were seeded into $60 \mathrm{~mm}$ tissue-culture dishes coated with rat-tail type I collagen (BD Discovery Labware, Bedford, MA) in bronchial epithelial growth medium (catalog no. CC-3170, Lonza) in a humidified HEPA-filtered cell culture incubator, supplemented with $5 \% \mathrm{CO}_{2}$. When the BECs reached $80 \%$ confluence, cells were passaged and seeded onto collagen-coated polyester 12-well transwell inserts with BEBM/DMEM. When the cell layer reached $100 \%$ confluence in the transwells, the culture method was shifted to the air-liquid interface (ALI) condition by removing the apical medium and maintain this condition for 10 days $[4,23]$. BECs were stimulated with or without IL-13 $(10 \mathrm{ng} / \mathrm{mL})$, purchased from Peprotech (Rocky Hill, NJ) and Mometasone Furoate (MF) at a concentration of $1 \mu \mathrm{M}$ (Sigma St Louis, MO).

Human fetal lung fibroblasts (HFL-1; lung, diploid, human, passage 3-7) were obtained from the American Type Culture Collection (Manassas, VA). HFL-1 cells were seeded into 24-well tissue culture plates at a density of $4 \times 10^{4}$ cells/well and cultured at $37{ }^{\circ} \mathrm{C}$ in a $5 \%$ $\mathrm{CO}_{2}$-humidified incubator in Ham's F12K medium (Sigma, St Louis, MO) containing $10 \%$ heat inactivated FBS. Cells were pretreated with recombinant human ITLN-1 (rhITLN-1) (ATGen, Gyeonggi-do, South Korea) at concentrations up to $500 \mathrm{ng} / \mathrm{mL}$ for $30 \mathrm{~min}$ and then further stimulated with a combination with TNF, IL-1 $\beta$, and IFN- $\gamma$ at $10 \mathrm{ng} / \mathrm{mL}$ (PeproTech, Rocky Hill, NJ). Cell-culture supernatants and extracts were harvested $24 \mathrm{~h}$ later.

\section{Western blot analysis for phospho-STAT1}

Protein samples $(10 \mu \mathrm{g})$ from HFL-1 were resolved on NuPage Novex 4-12\% Bis-Tris gel (Thermo Fisher Scientific, MA) electrophoresis, transferred, and immunoprobed with mouse monoclonal antibody for p-STAT1, total STAT1 (t-STAT1) (1:1000 and 1:500 respectively, Cell Signaling Technologies Inc. MA). Alkaline phosphatase conjugated secondary antibody (Thermo Fisher Scientific) was followed by Chemiluminescent detection (ChemiDoc XRD-J Bio-Rad Laboratories, Inc., CA). Densitometry was performed using the Quantity One (BioRad) and p-STAT1 indexed to t-STAT1.

\section{Statistical analysis}

Variables were checked for normality of distribution. As the majority of data were not normally distributed, data were analyzed using nonparametric tests. The Kruskal-Wallis version of the Wilcoxon rank sum test was used to compare overall differences among the groups (the overall $p$ value). When the overall $\mathrm{p}$ value was $<0.05$, intergroup comparisons were done using the Wilcoxon test for multiple comparisons. All other normal distributed data were analyzed using paired $t$ tests compared control and stimulated responses. $\mathrm{p}$ values $<0.05$ were considered significant. Linear regression analysis was used to determine the correlation with Type-2 related parameters and ITLN-1 mRNA. The statistical software used was the JMP version 10 (SAS Institute, Cary, NC).

\section{Results}

\section{Subjects}

Thirteen ST-Asthma, 18 SN-Asthma, 13 D-CON and 17 COPD subjects underwent bronchoscopic airway brushing (Table 1). D-CON and COPD were older than ST or SN-Asthma subjects ("p $<0.0001$, *** $p<0.05)$. FEV1/ FVC and \%FEV1 in COPD were lower than in D-CON, $\mathrm{ST}$ and SN-Asthma ("p $<0.0001)$. FeNO was significantly higher in SN-Asthma than the other groups ("p $<0.0001$ ). Mean ICS dose are represented Beclometasone dipropionate (BDP) equivalent dose. Two subjects were using OCS (predonisolone $5 \mathrm{mg} /$ day). Not all subject's cells were available for every experiments due to the limited numbers of epithelial cells obtained at the time of brushing. Table 2 includes 5 ST and 13 SN-Asthma who underwent BAL. FeNO in SN-Asthma tended to higher than ST-Asthma but this was not significant $(\mathrm{p}=0.08)$. Blood sample was collected from 6 ST-Asthma, 10 SN-Asthma and 8 healthy controls. (Table 3). FeNO in SN-Asthma were significantly higher than SN-Asthma (** $\mathrm{p}<0.01)$. 
ITLN-1 mRNA expression in freshly isolated BECs and correlation with Type- 2 related parameters in steroid naïve asthma

The mean counts of freshly isolated BECs from all the subjects were $4.4 \pm 0.6 \times 10^{5}$ from distal, and $4.9 \pm 1.1 \times 10^{5}$ from proximal ( 4 brushes each) brushings. They were over $90 \%$ pure and $80 \%$ viable. ITLN-1 mRNA expression in freshly isolated BECs was significantly higher in the SNAsthma group than in the other groups in both distal and proximal airway samples (overall $\mathrm{p}<0.0001$ ). There were no differences in ITLN-1 mRNA levels between distal and proximal samples among the groups (Fig. 1). Positive correlations were seen between ITLN-1 mRNA expression in the distal BECs and FeNO and IgE in SN-Asthma patients (Fig. 2a: $\mathrm{r}=0.84, \mathrm{p}<0.0001, \mathrm{~N}=16$ and $\mathrm{b}: \mathrm{r}=0.79$ $\mathrm{p}=0.0002, \mathrm{~N}=16$, respectively). ITLN-1 mRNA was also positively correlated with iNOS, CCL26, periostin and DPP4 mRNA (Fig. 2c-f), respectively. ITLN-1 mRNA and peripheral blood eosinophil numbers were marginally correlated $(\mathrm{r}=0.49 \mathrm{p}=0.0556, \mathrm{~N}=16$, data not shown). In proximal BECs, ITLN-1 mRNA was also correlated with FeNO, iNOS, CCL26 and periostin mRNA. In contrast, in ST-Asthma, ITLN-1 mRNA expression was low and there were no correlations with any Type- 2 related parameters (Additional file 1: Table S1).

\section{ITLN-1 appears to be primarily expressed by goblet cells}

Immunostaining of a small number of distal airway biopsy sections indicated that ITLN-1 protein was strongly expressed in goblet cells and weakly in brush border. Figure 3a shows representative staining from 3 SN-Asthma subjects stained with ITLN-1 antibody and isotype control IgG (Fig. 3b). Figure 3c (ITLN-1) and d (IgG) were representative staining from 3 ST-Asthma

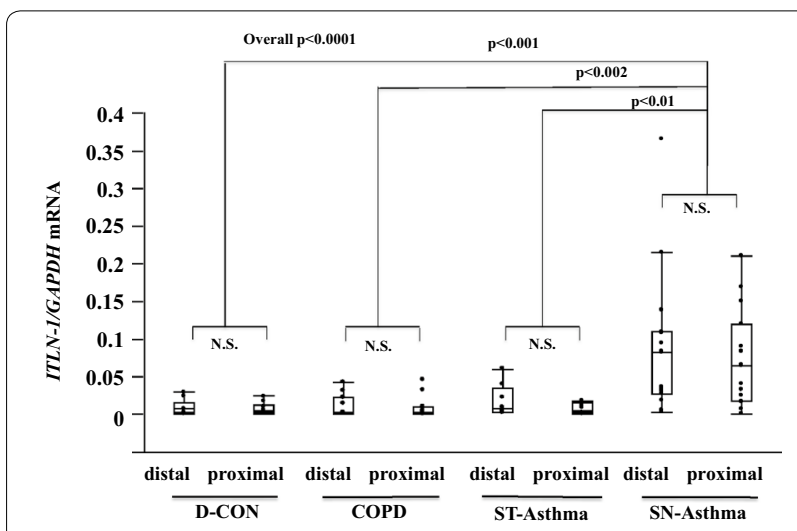

Fig. 1 ITLN-1 mRNA expression in freshly isolated BECs from each group by qPCR. ITLN-1 mRNA was significantly enhanced in freshly isolated distal and proximal BECs in SN-Asthma $(p<0.01)$ compared with other groups. Intergroup comparisons were done using the Wilcoxon test for multiple comparisons subjects after ICS (mometasone furoate; MF) treatment. Figure $3 \mathrm{c}$ and $\mathrm{d}$ was same subject with Fig. 3a, b after ICS treatment. Unfortunately, there were not enough biopsies of sufficient quality to evaluate differences among groups. Figure 3e shows ITLN-1 mRNA expressions in freshly isolated BECs samples in series of before and after ICS (MF) treatment in 6 samples from 3 subjects. Closed markers represent distal BECs and open diamonds are proximal BECs. ITLN-1 mRNA in both distal and proximal BECs significantly decreased after ICS treatments.

\section{ITLN-1 protein in BAL and serum}

ITLN-1 was detected in BAL and higher in SN-Asthma than ST-Asthma cases, although the concentrations were very low (Fig. 4a). In contrast, ITLN-1 was easily detected in serum in ST and SN-Asthma cases. Unexpectedly, there was no difference between the ST and SN-Asthma groups $(\mathrm{p}=0.21)$ in Fig. 4b. Serum and BALF albumin concentration were determined by ELISA. The ratio of ITLN-1/albumin was significantly higher in SN-Asthma (Additional file 2: Figure S1).

\section{ITLN-1 mRNA and protein is induced by IL-13 in primary cultured BECs}

ITLN-1 mRNA expression and protein were measured with or without IL-13 stimulation $(10 \mathrm{ng} / \mathrm{mL})$ in primary human BEC derived from both ST and SN-Asthma cultured in ALI. ITLN-1 mRNA expression and protein were significantly enhanced by IL-13 stimulation (Fig. 5a, b). However, amount of ITLN-1 mRNA was very low compared with freshly isolated BECs. Interestingly, ITLN-1 protein was detected only in apical supernatant. There were no differences in ITLN-1 mRNA or protein expression between SN-Asthma and ST-Asthma groups after IL-13 stimulation (Additional file 3: Figure S2a, b). Figure 5c and d show the inhibition effect of MF for induced ITLN-1 mRNA and protein by IL-13. MF inhibited IL-13 induced ITLN-1 mRNA significantly, and modest inhibition effect for ITLN-1 protein. ITLN-1 mRNA expression was significantly enhanced by IL-13 stimulation normalized by other housekeeping genes (B2M and RPLPO) (Additional file 4: Figure S3)

\section{CXCL10 mRNA and protein expression in HFL-1 cells is inhibited by ITLN-1}

To determine whether the Type- 2 associated ITLN-1 could functionally inhibit Type-1 associated inflammation, CXCL10 expression was induced by the combination of TNF, IL1 $\beta$ and IFN- $\gamma$ (Cytomix) in HFL- 1 cells and the inhibitory effects of ITLN-1 was evaluated (Fig. 6a, b). ITLN-1 (500 ng/mL) alone did not affect CXCL10 expression or production. However, ITLN-1 pretreatment $(30 \mathrm{~min})$ reduced Cytomix-induced CXCL10 mRNA and protein in a concentration-dependent 

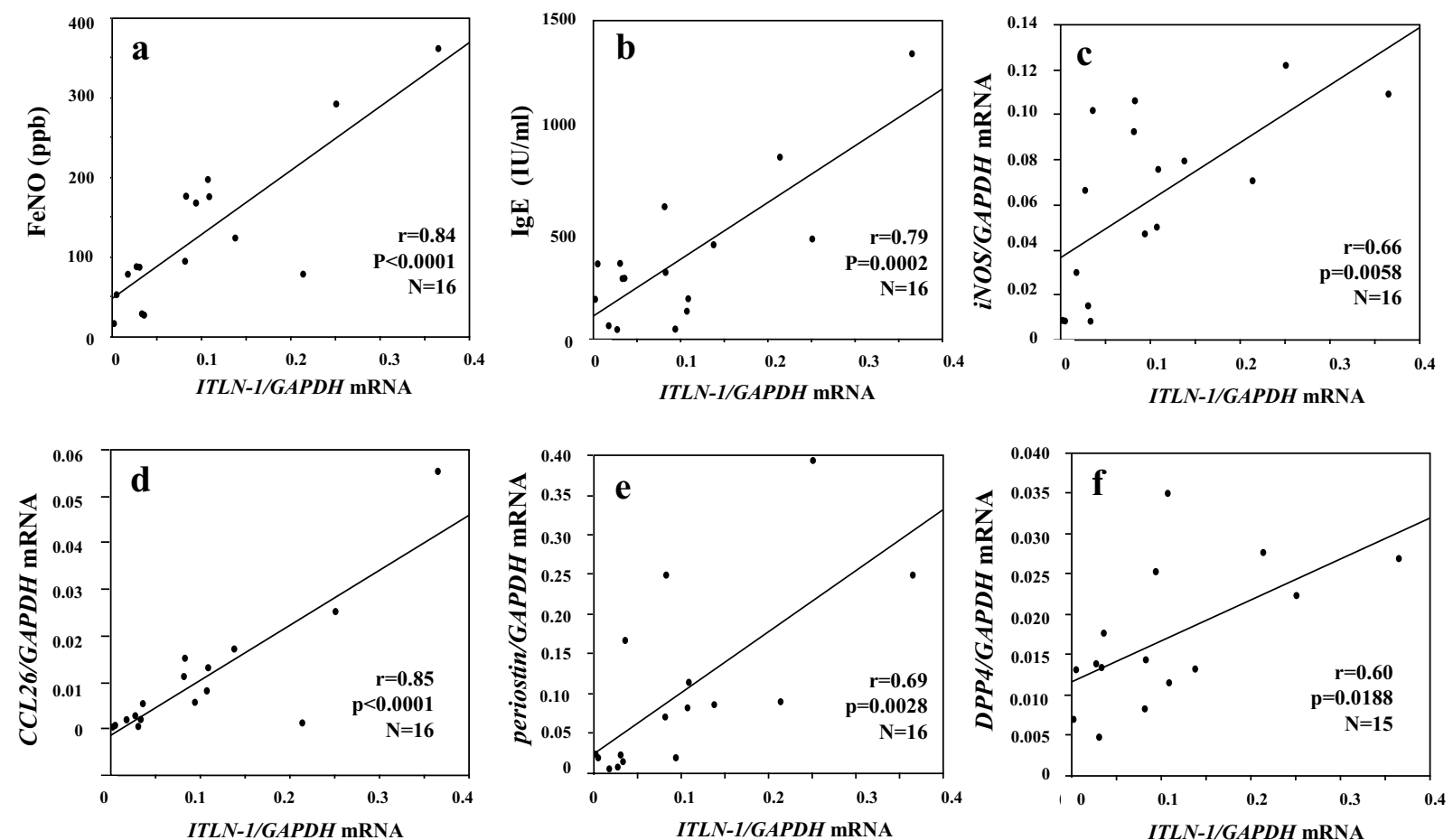

Fig. 2 Correlation between ITLN-1 mRNA and Type-2 related parameters in the distal airways in SN-Asthma patients. ITLN-1 mRNA showed significant correlation with FeNO (a $r=0.84, p<0.0001)$, $\lg E(\mathbf{b} r=0.79, p=0.0002)$ iNOS ( $\mathbf{c} r=0.66, p=0.0058)$, CCL26 (d $r=0.85, p<0.0001)$, periostin $(\mathbf{e}, r=0.69, p=0.0028)$ and DPP4 $(\mathbf{f}, r=0.60, p=0.0188)$ mRNA, respectively

manner. To investigate intracellular signal transduction, we examined STAT1 as a signal transduction pathway of IFN- $\gamma$. ITLN-1 decreased cytomix induced p-STAT1 at 5 and $15 \min (\mathrm{p}=0.0006, \mathrm{p}=0.0063$, respectively), supporting an inhibitory effect on this pathway.

\section{Discussion}

In this study, ITLN-1 was induced by IL-13 and mainly expressed in goblet cells of the distal and proximal airways in SN-Asthma patients. In the SN-Asthma group, ITLN-1 mRNA correlated with FeNO, IgE, iNOS, CCL26, periostin and DPP4 mRNA, all Type- 2 related parameters. Finally, our results suggest that ITLN-1 might lead to Type-2-bias by attenuating IFN- $\gamma$ signaling.

Kupermann et al. reported that ITLN-1 increased in an asthma model and in BECs from asthma subjects [17]. Similar to our data (Fig. 5a, b), Zen et al. showed that ITLN-1 expression increased in NHBE cells stimulated by IL-13 and in the lungs of mice after intranasal IL-13 administration and found ITLN-1 among the induced genes [24]. Gu et al. reported that ITLN-1 is required for expression of IL-13-induced monocyte chemotactic protein (MCP)-1 and -3 in lung epithelial cells and promotes allergic airway inflammation [25]. Thus, ITLN-1 appears to be strongly related to Type- 2 inflammation in vitro and in vivo. However, no reports have compared ITLN-1 with other asthma biomarkers or revealed its function in human asthma.

In this study, ITLN-1 mRNA significantly correlated with FeNO and serum IgE, as well as iNOS, CCL26, periostin and DPP4 mRNA in SN-Asthma, as these genes are known to be induced in BECs stimulated with IL-13. Kerr et al. showed that ITLN-1 in sputum is significantly higher in eosinophil-high groups, supporting an association of ITLN-1 with Type-2-high asthma [18]. Immunostaining showed that ITLN-1 protein was expressed in BECs, and suggested it was particularly expressed in goblet cells. The expression levels closely resembled those in intestinal epithelial cells published in previous reports $[15,18]$.

As Fig. 1 shows, ITLN-1 mRNA expression was significantly higher in freshly isolated BECs from the SNAsthma group than in the other groups, we hypothesized that ITLN-1 in BAL or more importantly in serum, could be a useful asthma biomarker. Comparing SN-Asthma and ST-Asthma groups only, BAL-ITLN-1 was detected 


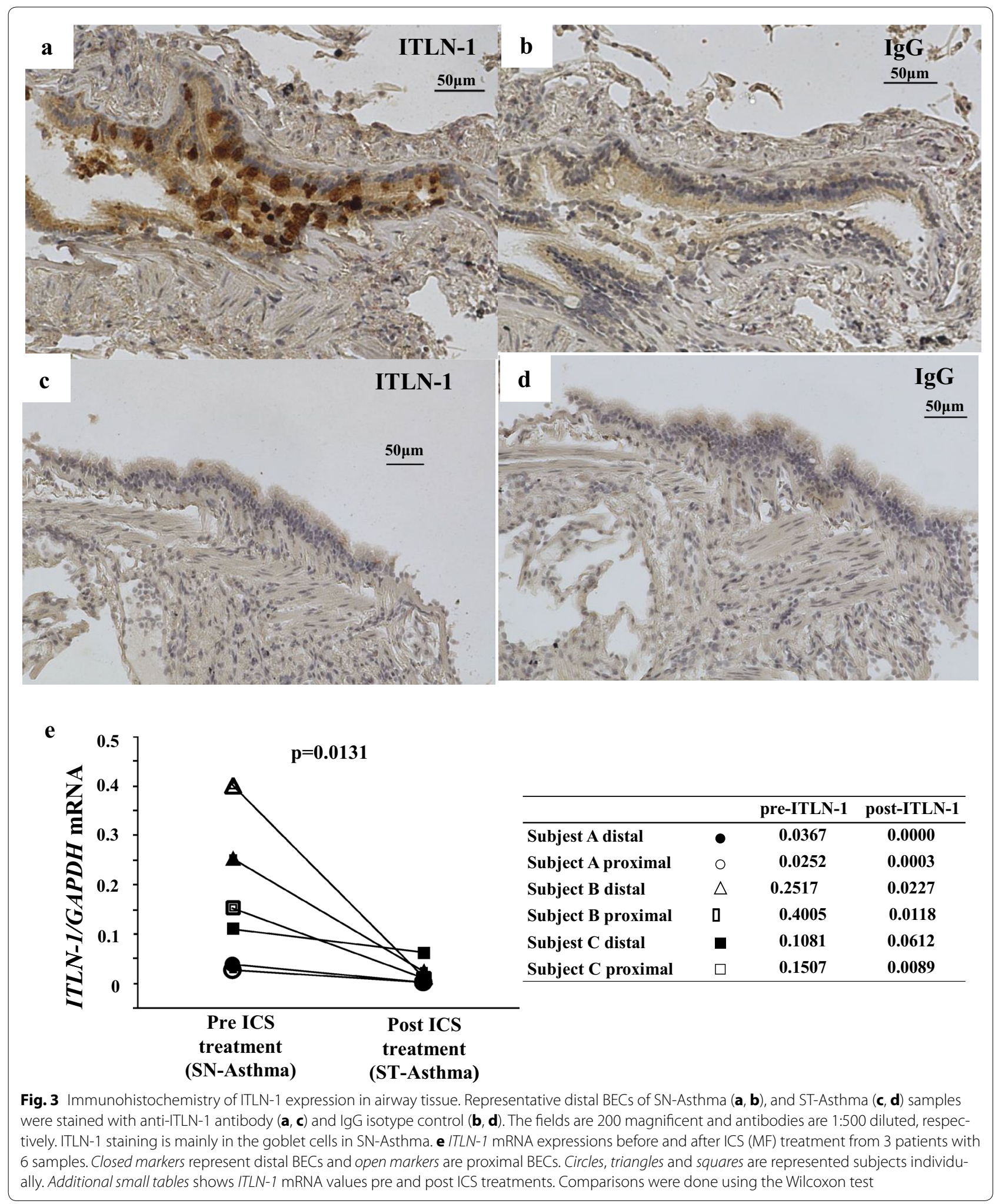




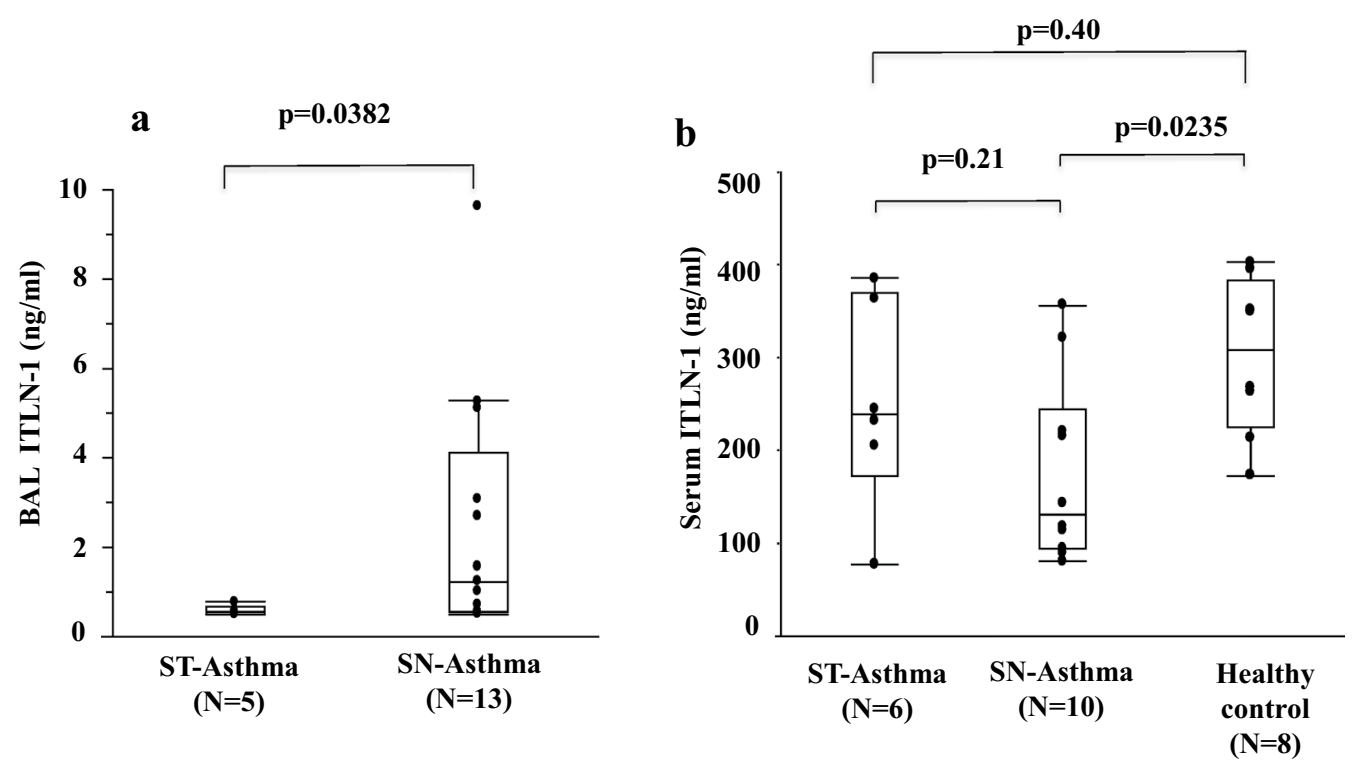

Fig. 4 ITLN-1 concentration in BAL and serum. a ITLN-1 concentration from BAL samples. ITLN-1 concentration was low (0.5-11.3, mean 2.4 ng/ $\mathrm{mL})$ but detectable by ELISA. ITLN-1 is higher in SN-Asthma $(n=13)$ than in ST-Asthma $(n=5)$ subjects $(p=0.0382$, Wilcoxon test). b Serum ITLN-1 concentration is abundant ( 77.3 to $402 \mathrm{ng} / \mathrm{mL}$, mean $236.5 \mathrm{ng} / \mathrm{mL}$ ). Serum ITLN-1 is no difference between ST and SN-Asthma subjects ( $p=0.21$ ) and ST-Asthma and healthy controls $(p=0.40)$. However, IT LN-1 is significantly higher than $S N$-Asthma $(p=0.0235)$. These comparisons were done using the Wilcoxon test

at low levels (range 0.5-9.6 $\mathrm{ng} / \mathrm{mL}$ ), and was significantly higher than in SN-Asthma as compared to ST-Asthma (Fig. 4a). However, BAL fluid collection is invasive, therefore we evaluated serum for ITLN-1. ITLN-1 was abundant in serum (range 77.3-385 $\mathrm{ng} / \mathrm{mL}$ ); but serum ITLN-1 was indistinguishable between ST-Asthma and SN-Asthma patients. This could be because systemic ITLN-1 may originate primarily from the intestinal tract or other organs as opposed to the airways. Moreover, ITLN-1 is expressed in goblet cells and mainly released into the lumens of the airways, such that the amount derived from the airways is not likely to reflect the serum ITLN-1. Thus, it does not appear that serum ITLN-1 will be a valid asthma biomarker.

As described earlier, ITLN-1 is a protective lectin against parasites and microorganisms. Suzuki et al. reported that ITLN-1 is a receptor of lactoferrin which helps to protect against infections [26]. It has been reported that ITLN-1 is expressed in the brush border of intestinal cells and binding of lactoferrin results in activation of signal transduction pathways that control infections. However, data on lactoferrin expression are controversial, with Kerr et al. also reporting increases in lactoferrin asthmatic sputum, while a recent gene array data suggested lower mRNA expression in asthma, particularly Type- 2 /severe asthma [18, 27]. Thus, further studies are needed to better understand the interactions between ITLN-1 and lactoferrin in asthma.

We also wished to examine the potential functions of ITLN-1 in the airway particularly in relation to Type- 1 inflammation. Previously, it was reported that ITLN-1 was one of an adipokine with anti-inflammatory effect [19]. CXCL10 (IP-10) is strongly induced by IFN- $\gamma$ and is a biomarker of Th1/Type-1 inflammation [28]. We hypothesized that ITLN-1 might skew cellular responses away from Type-1 pathways. Thus, we investigated whether rhITLN-1 could inhibit CXCL10 expression after stimulation with cytomix. ITLN-1 significantly inhibited cytomix induced CXCL10 in HFL-1 cells in a concentration-dependent manner, accompanied by a decrease in phosphorylation of STAT1. These results suggest that ITLN-1 could contribute to a Type-2-high bias in asthmatic airways.

The study limitations include the clinical/observational nature of the study which did not include specific bronchodilator responsiveness testing or methacholine challenge to confirm the asthma diagnosis, particularly 


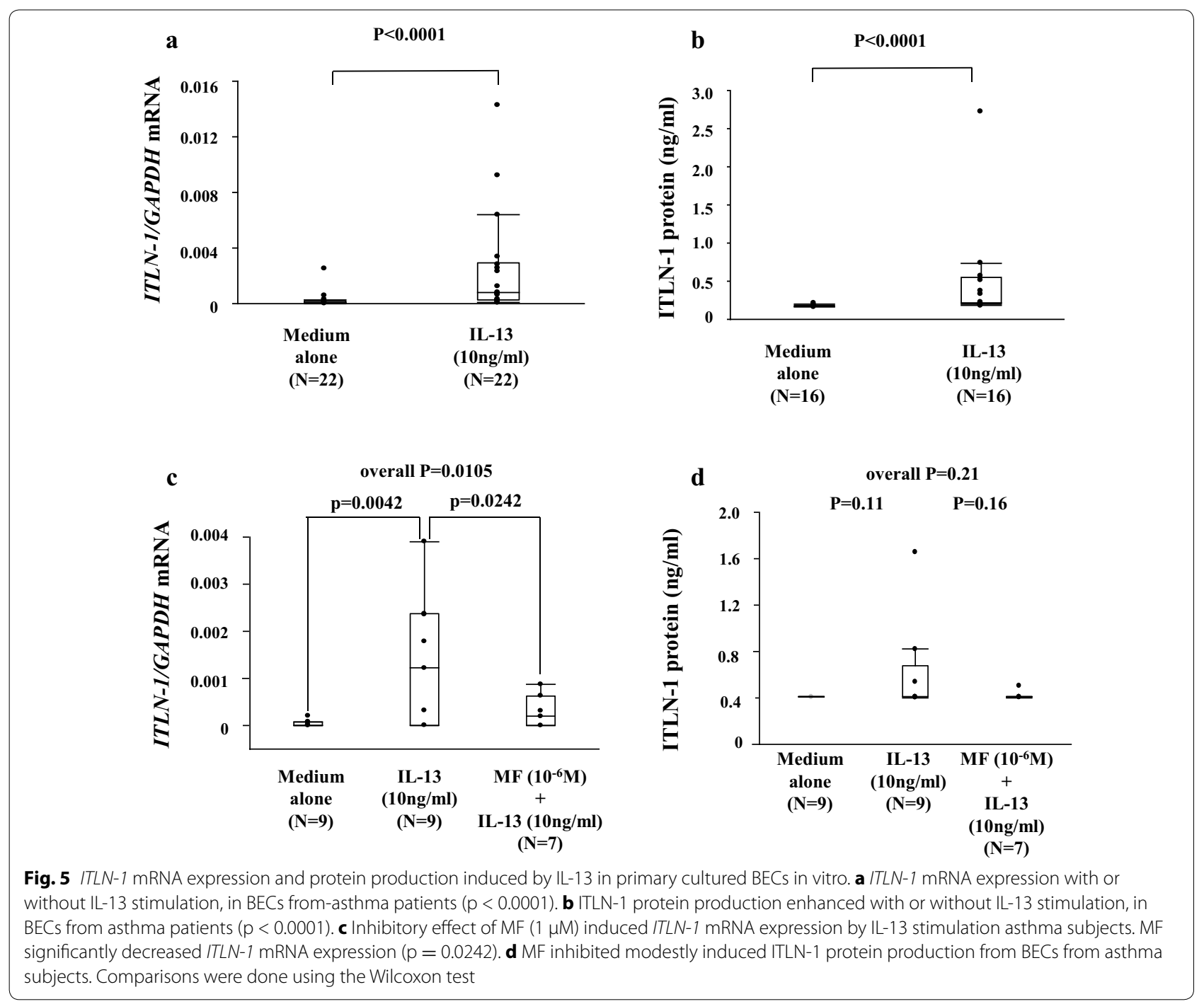

in the mild steroid naïve patients. However, despite this lack of objective data, differences in epithelial ITLN-1 expression were apparent on the basis of steroid treatment and in relation to known Type- 2 related parameters. We also lacked a true healthy control group. However, it is difficult to perform bronchoscopies in healthy individuals in Japan. Finally, these studies were done as add-on research studies to clinically indicated bronchoscopies in all patients. Therefore, the availability of BAL and tissue samples was limited to a small number of patients.

\section{Conclusions}

ITLN-1 is expressed in untreated asthmatic bronchial epithelial cells, particularly in goblet cells, in association with Type- 2 related parameters. However, it appears to be suppressed by corticosteroids in vivo, and epithelial ITLN-1 does not appear to contribute substantially to serum 


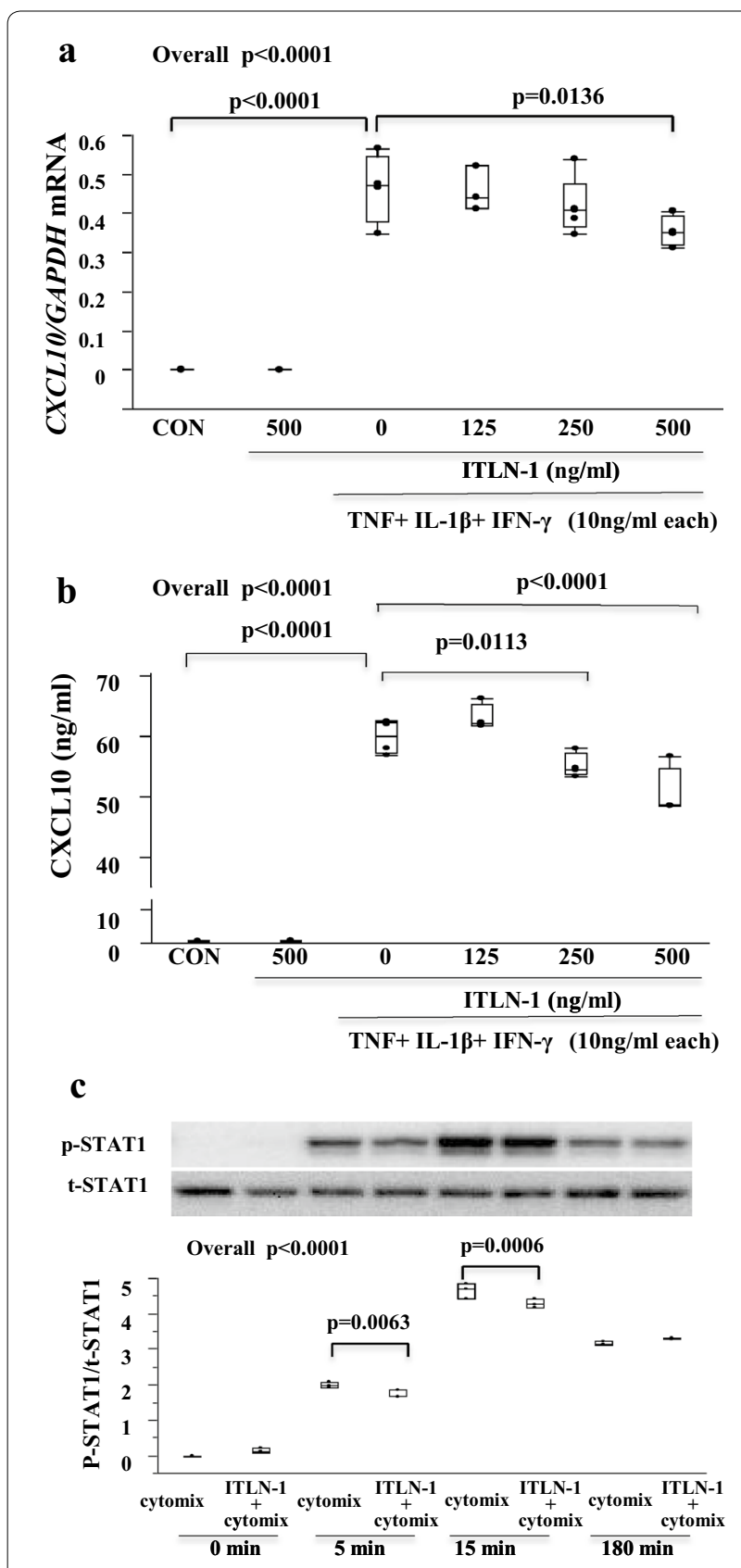

Fig. 6 Expression of CXCL10 stimulated by TNF, IL-1 $\beta$ and IFN- $\gamma$ and inhibition effect by pre-incubated ITLN-1. 30 min pre-incubated by ITLN-1 inhibited expression of CXCL10 mRNA (a) and CXCL10 protein (b) in HFL-1 stimulated by cytomix (TNF, IL-1 3 and IFN- $\gamma, 10 \mathrm{ng} / \mathrm{mL}$ each). a ITLN-1 at a concentration of $500 \mathrm{ng} / \mathrm{mL}$ inhibited cytomix induced $C X C L 10$ mRNA $(p=0.0136)$. b ITLN-1 at a concentration of 250 and $500 \mathrm{ng} / \mathrm{mL}$ inhibited cytomix induced CXCL10 protein ( $p<0.0001$ and $p=0.0113$, respectively). c Phospho ( $p$ )-STAT1/ total (t)-STAT1 level stimulated by cytomix and pre-incubated ITLN-1 in HFL-1. Phospho (p)-STAT1/t-STAT1 level was increased at 5 and $15 \mathrm{~min}, 30 \mathrm{~min}$ pre-treated ITLN-1 was signify inhibited phosphorylation of STAT- 1 ( $p=0.0006$ at $15 \mathrm{~min}$ and $p=0.0063$ at $5 \mathrm{~min}$ ) levels, making it unsuitable as a Type- 2 asthma biomarker. Its true role in asthma requires further study, perhaps in association with lactoferrin, but it has the potential to further skew inflammation away from Type- 1 and towards a Type-2 process.

\section{Additional files}

Additional file 1: Table S1. Correlation between ITLN-1 mRNA and Type-2 related parameters in the SN or ST-Asthma patients.

Additional file 2: Figure S1. Serum and BALF albumin concentration and BALF ITLN-1 ratio to BALF albumin.

Additional file 3: Figure S2. ITLN-1 mRNA expression and protein production induced by IL-13 in primary cultured BECs in vitro.

Additional file 4: Figure S3. ITLN-1 mRNA expression normalized by $B 2 M$ and RPLPO as housekeeping genes induced by IL-13 in primary cultured BECs in vitro.

\section{Abbreviations}

ITLN-1: intelectin-1; rhITLN-1: recombinant human ITLN-1; BECs: bronchial epithelial cells; FeNO: fractional exhaled nitric oxide; DPP4: dipeptidyl peptidase-4; iNOS: inducible nitric oxide synthase; CCL26: chemokine ligand 26; D-CON: disease control; ICS: inhaled corticosteroid; OCS: oral corticosteroid; SN-Asthma: ICS-naïve bronchial asthma; ST-Asthma: ICS-treated bronchial asthma; TBLB: transbronchial lung biopsy; EBB: endobronchial biopsy; BAL: bronchial alveolar lavage; ALI: air-liquid interface; HFL-1: human fetal fibroblasts; CXCL10: C-X-C motif chemokine 10; STAT1: signal transducer and activator of transcription 1.

\section{Authors' contributions}

TW, KC, TS, TR, RK, YN, RA, YH, and AT carried out sampling BECs and PCR studies, and drafted the manuscript. TW, KC, YH and TS carried out the immunoassays. TW, KC, YS, SW and YI participated in the design of the study and performed the statistical analysis. TW, KC, TF, SW and YI conceived of the study, and participated in its design and coordination and helped to draft the manuscript. All authors read and approved the final manuscript.

\section{Author details}

${ }^{1}$ Department of Pulmonary Medicine and Clinical Immunology, Dokkyo Medical University School of Medicine, Tochigi, Japan. ${ }^{2}$ Dokkyo Medical University School of Medicine, 880 Kitakobayashi Mibumachi, Shimotsugagun, Tochigi 321-0293, Japan. ${ }^{3}$ Pulmonary Allergy and Critical Care Medicine, Department of Medicine, University of Pittsburgh, 3459 Fifth Ave., Pittsburgh, PA 15213, USA.

\section{Acknowledgements}

We thank Reiko Komura and Kazumi Okazaki, who measured FeNO.

\section{Competing interests}

The authors declare that they have no competing interests.

\section{Availability of data and materials}

Datasets and materials are available on request by contacting the corresponding author Kazuyuki Chibana (kchibana@dokkyomed.ac.jp).

\section{Consent for publication}

Not applicable.

\section{Ethics approval and consent to participate}

This study was approved by the Ethics Committee of Dokkyo Medical University School of Medicine (hop-m22095). Written informed consent was 
obtained from all participants to perform the procedure and utilize extra tissue/cells for research purposes.

\section{Funding}

This study was by a Dokkyo Medical University, Young Investigator Award (No. 2013-16).

\section{Publisher's Note}

Springer Nature remains neutral with regard to jurisdictional claims in published maps and institutional affiliations.

Received: 23 January 2017 Accepted: 19 July 2017

Published online: 01 August 2017

\section{References}

1. Wenzel S, Castro M, Corren J, Maspero J, Wang L, Zhang B, Pirozzi G, Sutherland ER, Evans RR, Joish VN, et al. Dupilumab efficacy and safety in adults with uncontrolled persistent asthma despite use of mediumto-high-dose inhaled corticosteroids plus a long-acting $\beta 2$ agonist: a randomised double-blind placebo-controlled pivotal phase $2 \mathrm{~b}$ doseranging trial. Lancet. 2016;388:31-44.

2. Asakura T, Ishii Y, Chibana K, Fukuda T. Leukotriene D4 stimulates collagen production from myofibroblasts transformed by TGF-beta. J Allergy Clin Immunol. 2004;114:310-5

3. Chibana K, Ishii Y, Asakura T, Fukuda T. Up-regulation of cysteinyl leukotriene 1 receptor by $\mathrm{IL}-13$ enables human lung fibroblasts to respond to leukotriene C4 and produce eotaxin. J Immunol. 2003;170:4290-5.

4. Chibana K, Trudeau JB, Mustovich AT, Hu H, Zhao J, Balzar S, Chu HW, Wenzel SE. IL-13 induced increases in nitrite levels are primarily driven by increases in inducible nitric oxide synthase as compared with effects on arginases in human primary bronchial epithelial cells. Clin Exp Allergy. 2008;38:936-46.

5. Yamamoto M, Tochino Y, Chibana K, Trudeau JB, Holguin F, Wenzel SE. Nitric oxide and related enzymes in asthma: relation to severity, enzyme function and inflammation. Clin Exp Allergy. 2012;42:760-8.

6. Zhao J, Maskrey B, Balzar S, Chibana K, Mustovich A, Hu H, Trudeau JB, O'Donnell V, Wenzel SE. Interleukin-13-induced MUC5AC is regulated by 15 -lipoxygenase 1 pathway in human bronchial epithelial cells. Am J Respir Crit Care Med. 2009:179:782-90.

7. Shiobara T, Chibana K, Watanabe T, Arai R, Horigane Y, Nakamura Y, Hayashi Y, Shimizu Y, Takemasa A, Ishii Y. Dipeptidyl peptidase-4 is highly expressed in bronchial epithelial cells of untreated asthma and it increases cell proliferation along with fibronectin production in airway constitutive cells. Respir Res. 2016;17:28

8. Takayama G, Arima K, Kanaji T, Toda S, Tanaka H, Shoji S, McKenzie AN, Nagai $\mathrm{H}$, Hotokebuchi T, Izuhara K. Periostin: a novel component of subepithelial fibrosis of bronchial asthma downstream of IL-4 and IL-13 signals. J Allergy Clin Immunol. 2006;118:98-104.

9. Corren J, Lemanske RF, Hanania NA, Korenblat PE, Parsey MV, Arron JR, Harris JM, Scheerens H, Wu LC, Su Z, et al. Lebrikizumab treatment in adults with asthma. New Engl J Med. 2011;365:1088-98.

10. Izuhara K, Conway SJ, Moore BB, Matsumoto H, Holweg CT, Matthews JG, Arron JR. Roles of periostin in respiratory disorders. Am J Respir Crit Care Med. 2016;193:949-56.

11. Brightling CE, Chanez P, Leigh R, O'Byrne PM, Korn S, She D, May RD, Streicher K, Ranade K, Piper E. Efficacy and safety of tralokinumab in patients with severe uncontrolled asthma: a randomised, double-blind, placebo-controlled, phase 2b trial. Lancet Respir Med. 2015;3:692-701.

12. Komiya T, Tanigawa Y, Hirohashi S. Cloning of the novel gene intelectin, which is expressed in intestinal paneth cells in mice. Biochem Biophys Res Commun. 1998:251:759-62.

13. Tsuji S, Uehori J, Matsumoto M, Suzuki Y, Matsuhisa A, Toyoshima K, Seya T. Human intelectin is a novel soluble lectin that recognizes galactofuranose in carbohydrate chains of bacterial cell wall. J Biol Chem. 2001;276:23456-63.

14. Pemberton AD, Knight PA, Gamble J, Colledge WH, Lee JK, Pierce M, Miller HR. Innate BALB/c enteric epithelial responses to Trichinella spiralis: inducible expression of a novel goblet cell lectin, intelectin-2, and its natural deletion in C57BL/10 mice. J Immunol. 2004;173:1894-901.
15. French AT, Knight PA, Smith WD, Pate JA, Miller HR, Pemberton AD. Expression of three intelectins in sheep and response to a Th2 environment. Vet Res. 2009;40:53

16. Tsuji S, Tsuura Y, Morohoshi T, Shinohara T, Oshita F, Yamada K, Kameda Y, Ohtsu T, Nakamura Y, Miyagi Y. Secretion of intelectin-1 from malignant pleural mesothelioma into pleural effusion. Br J Cancer. 2010;103:517-23.

17. Kuperman DA, Lewis CC, Woodruff PG, Rodriguez MW, Yang YH, Dolganov GM, Fahy JV, Erle DJ. Dissecting asthma using focused transgenic modeling and functional genomics. J Allergy Clin Immunol. 2005;116:305-11.

18. Kerr SC, Carrington SD, Oscarson S, Gallagher ME, Solon M, Yuan S, Ahn JN, Dougherty RH, Finkbeiner WE, Peters MC, Fahy JV. Intelectin-1 is a prominent protein constituent of pathologic mucus associated with eosinophilic airway inflammation in asthma. Am J Respir Crit Care Med. 2014;189:1005-7.

19. Zhong X, Li X, Liu F, Tan H, Shang D. Omentin inhibits TNF-alpha-induced expression of adhesion molecules in endothelial cells via ERK/NF-kappaB pathway. Biochem Biophys Res Commun. 2012;425:401-6.

20. Bhushan B, Homma T, Norton JE, Sha Q, Siebert J, Gupta DS, Schroeder JW Jr, Schleimer RP. Suppression of epithelial signal transducer and activator of transcription 1 activation by extracts of Aspergillus fumigatus. Am J Respir Cell Mol Biol. 2015;53:87-95.

21. Matsukura S, Kokubu F, Kurokawa M, Kawaguchi M, leki K, Kuga H, Odaka M, Suzuki S, Watanabe S, Takeuchi H, et al. Synthetic double-stranded RNA induces multiple genes related to inflammation through Toll-like receptor 3 depending on NF-kappaB and/or IRF-3 in airway epithelial cells. Clin Exp Allergy. 2006;36:1049-62.

22. Yoshizawa T, Hammaker D, Sweeney SE, Boyle DL, Firestein GS. Synoviocyte innate immune responses: I. Differential regulation of interferon responses and the JNK pathway by MAPK kinases. J Immunol. 2008;181:3252-8.

23. Chu HW, Balzar S, Seedorf GJ, Westcott JY, Trudeau JB, Silkoff P, Wenze SE. Transforming growth factor- $\beta 2$ induces bronchial epithelial mucin expression in asthma. Am J Pathol. 2004:165:1097-106.

24. Zhen G, Park SW, Nguyenvu LT, Rodriguez MW, Barbeau R, Paquet AC, Erle DJ. IL-13 and epidermal growth factor receptor have critical but distinct roles in epithelial cell mucin production. Am J Respir Cell Mol Biol. 2007;36:244-53.

25. Gu N, Kang G, Jin C, Xu Y, Zhang Z, Erle DJ, Zhen G. Intelectin is required for IL-13-induced monocyte chemotactic protein-1 and -3 expression in lung epithelial cells and promotes allergic airway inflammation. Am J Physiol Lung Cell Mol Physiol. 2010;298:L290-6.

26. Suzuki YA, Shin K, Lonnerdal B. Molecular cloning and functional expression of a human intestinal lactoferrin receptor. Biochemistry. 2001:40:15771-9.

27. Modena BD, Tedrow JR, Milosevic J, Bleecker ER, Meyers DA, Wu W, Bar-Joseph Z, Erzurum SC, Gaston BM, Busse WW, et al. Gene expression in relation to exhaled nitric oxide identifies novel asthma phenotypes with unique biomolecular pathways. Am J Respir Crit Care Med. 2014:190:1363-72

28. Yoshikawa M, Wada K, Yoshimura T, Asaka D, Okada N, Matsumoto K, Moriyama $\mathrm{H}$. Increased CXCL10 expression in nasal fibroblasts from patients with refractory chronic rhinosinusitis and asthma. Allergol Int. 2013:62:495-502

\section{Submit your next manuscript to BioMed Central and we will help you at every step:}

- We accept pre-submission inquiries

- Our selector tool helps you to find the most relevant journal

- We provide round the clock customer support

- Convenient online submission

- Thorough peer review

- Inclusion in PubMed and all major indexing services

- Maximum visibility for your research

Submit your manuscript at www.biomedcentral com/submit
C BioMed Central 